\title{
Study of mass attenuation coefficients, effective atomic numbers and electron densities of carbon steel and stainless steels
}

\author{
V.P. SINGH $^{1,2}$, N.M. BADIGER ${ }^{1}$
}

(Manuscript received 21 February 2013, accepted 19 April 2013)

\begin{abstract}
The mass attenuation coefficient $(\mu / \rho)$, effective atomic number $\left(Z_{\text {Pleff }}\right)$ and effective electron density $\left(\mathrm{N}_{\mathrm{eff}}\right)$ of carbon steel and stainless steels were computed by using the WinXcom program. The $\mu / \rho$ values are higher in the photo-electric absorption and pair production region and approximately constant in the Compton scattering region. The variation in $\mu / \rho$ values is explained by partial interaction processes. The effective atomic numbers of the steels were also calculated by the Auto- $Z_{\text {eff }}$ program. The $Z_{\text {Pleff }}$ values of the steels were compared with experimental results for available energies and were found to be in good agreement. SS304L shows equal values of effective atomic number by both the methods in the region of interest. The electron densities of the selected steels are observed to be equal and approximately constant in the Compton scattering region.
\end{abstract}

Keywords: steel / effective atomic number / mass attenuation coefficient / Auto- $Z_{\text {eff }}$

\section{Introduction}

The application of gamma radiation has been increased in science and technology; industry, medicine, agriculture, petroleum plants, energy sectors, etc. In practice, gamma-ray sources above $200 \mathrm{keV}$ up to $1500 \mathrm{keV}$ are widely used in radiography, archaeometry, chemotherapy, medical diagnosis and Compton scatter images (Hubbel et al., 1975). Gamma rays can be absorbed in high-density materials such as lead, tungsten, concretes and building materials. Gamma-ray interaction is dependent upon the photon energy and atomic number of the elements of the composite materials. The absorption of gamma rays is represented by the mass attenuation coefficients, molecular cross-sections, atomic crosssections, effective atomic numbers and electron densities. The linear attenuation coefficient for a material depends on the incident photon energy, atomic number and density. The attenuation coefficient is defined as the probability of radiation interacting with a material per unit path length (Woods, 1982). Hine (1952)

\footnotetext{
Department of Physics, Karnatak University, Dharwad 580003, India.

2 Permanent address: Health Physics Section, Kaiga Atomic Power Station-3\&4, NPCIL, Karwar, 581400, India, email: kudphyvps@rediffmail.com
} 
pointed out that in the study of gamma interaction with complex media, the atomic number of the complex media cannot be represented by a single value for the entire energy, similar to the elements. This is due to the fact that gamma-ray interaction is dependent on the atomic number. Thus, a term called the "effective atomic number" should be defined for such complex materials. The effective atomic number is a convenient parameter for evaluation of gamma-ray interaction with a material. The effective atomic number can provide an initial estimation of the chemical compositions of the complex, neutron/gamma-ray shielding effectiveness and other parameters. In general, the effective atomic number of inorganic compounds, metals and alloys is large (Singh and Badiger, 2012a, 2012b), while it is small for organic substances (Singh and Badiger, 2013). The other important quantity for interaction is the effective electron number or electron density, as it is defined as the electrons per unit mass of the absorber.

The mass attenuation coefficient, effective atomic number and electron density are the gamma-ray interaction parameters to describe shielding effectiveness. Mass attenuation coefficients of elements are taken from standard tables (Berger and Hubbell, 1987/99) developed as the XCOM program for theoretical values for mass attenuation coefficients and cross-sections for various elements, compounds and mixtures at energies of $1 \mathrm{keV}$ to $100 \mathrm{GeV}$. The XCOM program was converted to the Windows platform and is known as WinXcom (Gerward et al., 2001, 2004), which is very useful for analysis.

Gamma-ray absorption is a vital area; various researchers have carried out studies for different types of materials. Effective atomic numbers of spin ice compounds, gaseous mixtures, alcohols, alloys, solid-state neutron track detectors, vitamins and composite mixtures have been obtained (İçelli et al., 2005; Bastug et al., 2010; Medhat, 2011; Demir et al., 2012; Singh and Badiger, 2012a, 2012b, 2013). Mass attenuation coefficients, effective atomic numbers and electron densities of thermoluminescent dosimetric compounds (Shivaliga et al., 2004) have also been obtained. The mass attenuation coefficients, and effective atomic and electron numbers for some alloys such as $\mathrm{Cr}, \mathrm{Fe}$ and $\mathrm{Ni}$ at different energies have been reported (Kaewkhao et al., 2008; Han et al., 2009). The effective atomic numbers of various alloys have been estimated (Prasad et al., 1998; El-Kateb et al., 2000; Murty et al., 2000; İçelli et al., 2005; Akkurt, 2007, 2009).

In the present work, we computed the mass attenuation coefficients, effective atomic numbers and electron densities of the carbon steel and stainless steels given in Table I. The compositions of the stainless steels ( 1 to 4$)$ are taken from the proceeding (Prasanna and Kumar, 2012) as supplied by the manufacturer. The theoretical values of the effective atomic numbers of these materials were 
TABLE I

Chemical composition of carbon steel and stainless steels.

\begin{tabular}{|c|c|c|c|c|c|c|c|c|c|c|c|c|}
\hline & \multicolumn{12}{|c|}{ Composition (weight percentage) } \\
\hline & $\mathrm{C}$ & $\mathrm{Al}$ & $\mathrm{Si}$ & $P$ & S & $\mathrm{Ti}$ & $\mathrm{Cr}$ & $\mathrm{Mn}$ & $\mathrm{Fe}$ & $\mathrm{Ni}$ & $\mathrm{Cu}$ & Mo \\
\hline Steel 1 & 0.4200 & 0.0490 & 0.2500 & 0.0210 & 0.1100 & 0.0019 & 1.0500 & 0.8900 & 97.0371 & 0.0600 & 0.0100 & 0.2000 \\
\hline Steel 2 & 0.3900 & 0.0360 & 0.2600 & 0.0140 & 0.0080 & 0.0014 & 0.0400 & 0.7900 & 98.4206 & 0.0200 & 0.0100 & 0.0100 \\
\hline Steel 3 & 1.0300 & 0.0340 & 0.3100 & 0.0150 & 0.0120 & 0.0026 & 1.4300 & 0.3200 & 96.7960 & 0.0300 & 0.0100 & 0.0100 \\
\hline Steel 4 & 0.0330 & 0.0400 & 0.2600 & 0.0200 & 0.0210 & 0.0013 & 0.8900 & 0.6600 & 98.5777 & 0.0300 & 0.0100 & 0.1600 \\
\hline CS516 & 0.3100 & 0.0000 & 0.1900 & 0.0100 & 0.0050 & 0.0000 & 0.0000 & 0.9800 & 98.3900 & 0.0000 & 0.0000 & 0.0000 \\
\hline SS410 & 0.1500 & 0.0000 & 1.0000 & 0.0400 & 0.0300 & 0.0000 & 11.2500 & 1.0000 & 85.7800 & 0.7500 & 0.0000 & 0.0000 \\
\hline SS316L & 0.0300 & 0.0000 & 0.5300 & 0.0400 & 0.0050 & 0.0000 & 16.0000 & 1.8500 & 68.2700 & 10.7700 & 0.0000 & 2.1200 \\
\hline SS304L & 0.0000 & 0.0000 & 0.0000 & 0.0000 & 0.0000 & 0.0000 & 18.0000 & 0.0000 & 74.0000 & 8.0000 & 0.0000 & 0.0000 \\
\hline
\end{tabular}

compared with available experimental values at $279.1 \mathrm{keV}, 661.6 \mathrm{keV}, 662 \mathrm{keV}$, $1115.5 \mathrm{keV}, 1173 \mathrm{keV}$ and $1332 \mathrm{keV}$, which show good agreement.

\section{Computational work}

Computation of the mass attenuation coefficients of the carbon steel and stainless steels was carried out by the mixture rule by using the WinXcom program for the energies $1 \mathrm{keV}$ to $100 \mathrm{GeV}$ (Gerward et al., 2001, 2004). Computation of the effective atomic numbers and electron densities of carbon steel and stainless steel by total gamma photon interaction was carried out by a practical formula (Manohara et al., 2008). The atomic numbers and atomic masses of the elements were taken from the atomic weights of the elements 2009, IUPAC (Michael and Tyler, 2011). The steel samples, steel 1 , steel 4, CS516 and SS316L, are unnormalized compositions of elements which have been normalized to 1 by the software for calculation. As the difference is insignificant, the impact on the results will be negligible.

\subsection{Mass attenuation coefficients}

The mass attenuation coefficient $(\mu / \rho)_{\text {steel }}$ is given by:

$$
(\mu / \rho)_{\text {steel }}=\sum_{i}^{n} w_{i}\left(\frac{\mu}{\rho}\right)_{i}
$$

where $\mathrm{w}_{\mathrm{i}}$ is the proportion by weight and $(\mu / \rho)_{\mathrm{i}}$ is the mass attenuation coefficient of the ith element. 
The quantity $\mathrm{w}_{\mathrm{i}}$ is given by:

$$
w_{i}=\frac{n_{i} A_{i}}{\sum_{j} n_{j} A_{j}}
$$

with the condition that

$$
\mathrm{w}_{1}+\mathrm{w}_{2}+\mathrm{w}_{3}+\ldots \ldots+\mathrm{w}_{\mathrm{i}}=1
$$

where $A_{i}$ is the atomic weight of the ith element and $n_{i}$ is the number of formula units in the compounds.

\subsection{Effective atomic numbers}

The effective atomic number $\left(\mathrm{Z}_{\text {Pleff }}\right)$ for total photon interaction is given by:

$$
Z_{\text {Pleff }}=\frac{\Sigma_{i} f_{i} A_{i}\left(\frac{\mu}{\rho}\right)_{i}}{\Sigma_{j} f_{j} \frac{A_{j}}{Z_{j}}\left(\frac{\mu}{\rho}\right)_{j}}
$$

where $f_{i}$ is the molar fraction in the mixture/alloys, $\mu$ is the linear attenuation coefficient, $\rho$ is density, $\mu / \rho$ is the mass attenuation coefficient, $A$ is the atomic weight, $\mathrm{Z}$ is the atomic number, and the ratio $\mathrm{A} / \mathrm{Z}$ between the atomic mass and the atomic number is approximately constant. Recently, a new software program has made the tedious calculations of the present work very easy. This software Auto$\mathrm{Z}_{\text {eff }}$ is freely available and the effective atomic numbers of soft tissue, brass, oleic acid, $\mathrm{CaSO}_{4}$ : Dy, water and alloys have been estimated (Taylor et al., 2012). The effective atomic numbers calculated by Auto- $Z_{\text {eff }}$ and experimental values demonstrated good agreement.

\subsection{Electron densities}

The electron density is given by $\mathrm{N}_{\text {eff }}=\mathrm{N}_{\mathrm{A}} \mathrm{Z} / \mathrm{A}$, which is generalized as:

$$
N_{\text {eff }}=N_{A} \frac{n Z_{\text {Pleff }}}{\Sigma_{i} n_{i} A_{i}}=N_{A} \frac{Z_{\text {Pleff }}}{\langle A\rangle}
$$

where $n_{i}$ is the number of atoms of the ith constituent element, $n$ is the total number of atoms and $\langle\mathrm{A}\rangle$ is the average atomic mass of the steel alloys. 


\section{Results and discussion}

Variation in mass attenuation coefficients for the selected alloys for the gammaray energy range $1 \mathrm{keV}$ to $100 \mathrm{GeV}$ is shown in Figure 1. Partial photon interaction with these steels is shown in Figure $2(\mathrm{a}-\mathrm{h})$ for understanding of the interaction processes (photo-electric absorption, Compton scattering and pair production) and their contribution. The effective atomic numbers and electron densities of the selected steels are shown in Figures 3 and 4, respectively, for the photon energies $10 \mathrm{keV}$ to $1 \mathrm{GeV}$. Gamma-ray interaction is based on photo-electric absorption, Compton scattering and pair production. Therefore, we studied separately the mass attenuation coefficients of steels for coherent, incoherent, photo-electric absorption and pair (nuclear/electron).

\subsection{Mass attenuation coefficients}

Variation in $(\mu / \rho)_{\text {steel }}$ with gamma photon energy is shown in Figure 1. From Figure 1 , it is clear that the $(\mu / \rho)_{\text {steel }}$ of the steels decreases with an increase in energy of the incident photon. It can be seen that the $(\mu / \rho)_{\text {steel }}$ values decrease sharply up to $100 \mathrm{keV}$ photon energy, further decrease very slowly in the photon energy region $100 \mathrm{keV}$ to $10 \mathrm{MeV}$, and afterward increase again and become constant. The $(\mu / \rho)_{\text {steel }}$ values of all the steels show one sharp peak at $7.11 \mathrm{keV}$. This peak is due to k-edge absorption for nickel and copper, which lies at the photon energies $8.3 \mathrm{keV}$ and $8.979 \mathrm{keV}$, respectively. At around $1 \mathrm{MeV}$ photon energy, the $(\mu / \rho)_{\text {steel }}$ values are found to be constant. Complete variation in $(\mu / \rho)_{\text {steel }}$ with photon energy for partial interaction processes is explained in the next sub-sections.

\subsubsection{Photo-electric absorption process}

From Figure $2(\mathrm{a}-\mathrm{h})$, it is observed that the values of $\mu_{\text {steel, photo }}$ decrease rapidly with an increase in incident photon energy for all the selected steels. Initially, at $1 \mathrm{keV}$, the value of $\mu_{\mathrm{m}}$ (photo) is very high; of the order of $10^{3}$, which decreases sharply. The values of $\mu_{\text {steel, photo }}$ of all the steels become negligible $\left(<10^{-3}\right)$ as photon energy reaches $100 \mathrm{keV}$. The reason for such variation may be due to dependency of the photo-electric cross-section on photon energy as $\mathrm{E}^{3.5}$. Hence, the $\mu_{\mathrm{m}}$ (photo) values decrease rapidly at low energy. This shows the dominance of photo-electric absorption in the low photon energy region.

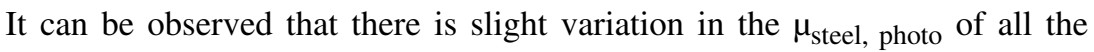
selected steels at particular photon energies. It is due to the reason that photoelectric absorption is dependent on the atomic numbers of the elements of the mixture or compounds as $Z^{4.5}$. The steels in our investigation contain different 


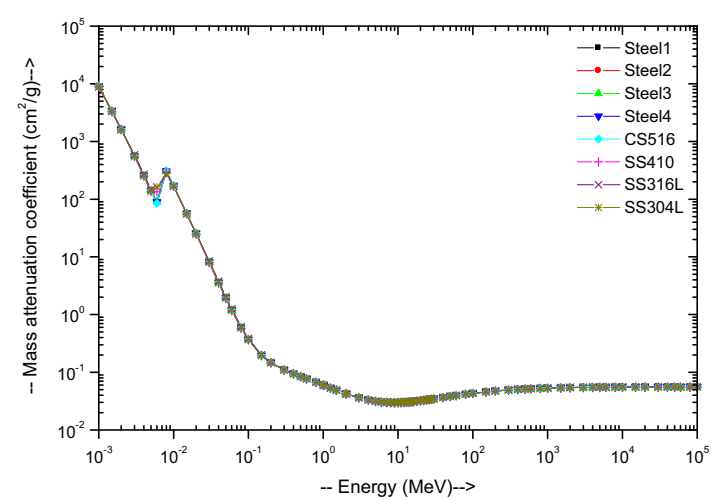

Figure 1 - Variation in mass attenuation coefficients of carbon steel and stainless steels with photon energy.

constituent elements $\left({ }_{6} \mathrm{C},{ }_{13} \mathrm{Al},{ }_{14} \mathrm{Si},{ }_{15} \mathrm{P},{ }_{16} \mathrm{~S},{ }_{22} \mathrm{Ti},{ }_{24} \mathrm{Cr},{ }_{25} \mathrm{Mn},{ }_{26} \mathrm{Fe},{ }_{28} \mathrm{Ni},{ }_{29} \mathrm{Cu}\right.$ and ${ }_{42} \mathrm{Mo}$ ) with different weight fractions or numbers of atoms, as given in Table I. The slight difference in the weight fraction of elements results in insignificant variation in $\mu_{\mathrm{m}}$ (photo) values.

\subsubsection{Compton scattering process}

From Figure $2(\mathrm{a}-\mathrm{h})$, it is observed that $\mu_{\text {steel, coherent }}$ decreases from $1 \mathrm{keV}$ very fast and becomes insignificant at around $1 \mathrm{MeV}$, whereas $\mu_{\text {steel, coherent }}$ increases from $1 \mathrm{keV}$ to $100 \mathrm{keV}$, thereafter decreases, and becomes insignificant at around $200 \mathrm{MeV}$ photon energy. For the combined effect of Compton scattering, it is observed that $\mu_{\text {steel, coherent }}$ initially increases from $1 \mathrm{keV}$ to $10 \mathrm{keV}$, thereafter becomes constant up to $100 \mathrm{keV}$ and, with a further increase in the incident photon energy, $\mu_{\text {steel, coherent }}$ decreases slowly up to $200 \mathrm{MeV}$. Further, it is also observed that $\mu_{\text {steel, coherent }}$ and $\mu_{\text {steel, photo }}$ values are equivalent at $20 \mathrm{keV}$. Above $20 \mathrm{keV}$ photon energy, it can be seen that Compton scattering is the dominant process of interaction up to $20 \mathrm{MeV}$ for all the selected steels. It can also be noted that the variation in $\mu_{\text {steel, coherent }}$ is slower compared with $\mu_{\text {steel, photo. }}$.

\subsubsection{Pair production process}

From Figure $2(\mathrm{a}-\mathrm{h})$, it can be observed that the mass attenuation coefficient values for the pair production process in the nuclear field, $\mu_{\text {steel, npp }}$, and electron field,

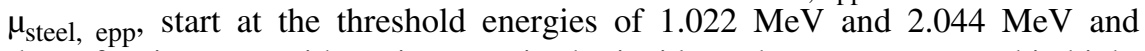
thereafter increase with an increase in the incident photon energy. In this highenergy region, a slight variation is again seen in $\mu_{\text {steel, pp }}$ due to their different chemical compositions at particular incident photon energies (Fig. 2 (a-h)). It may 
PHOTON INTERACTION WITH CARBON STEEL AND STAINLESS STEELS
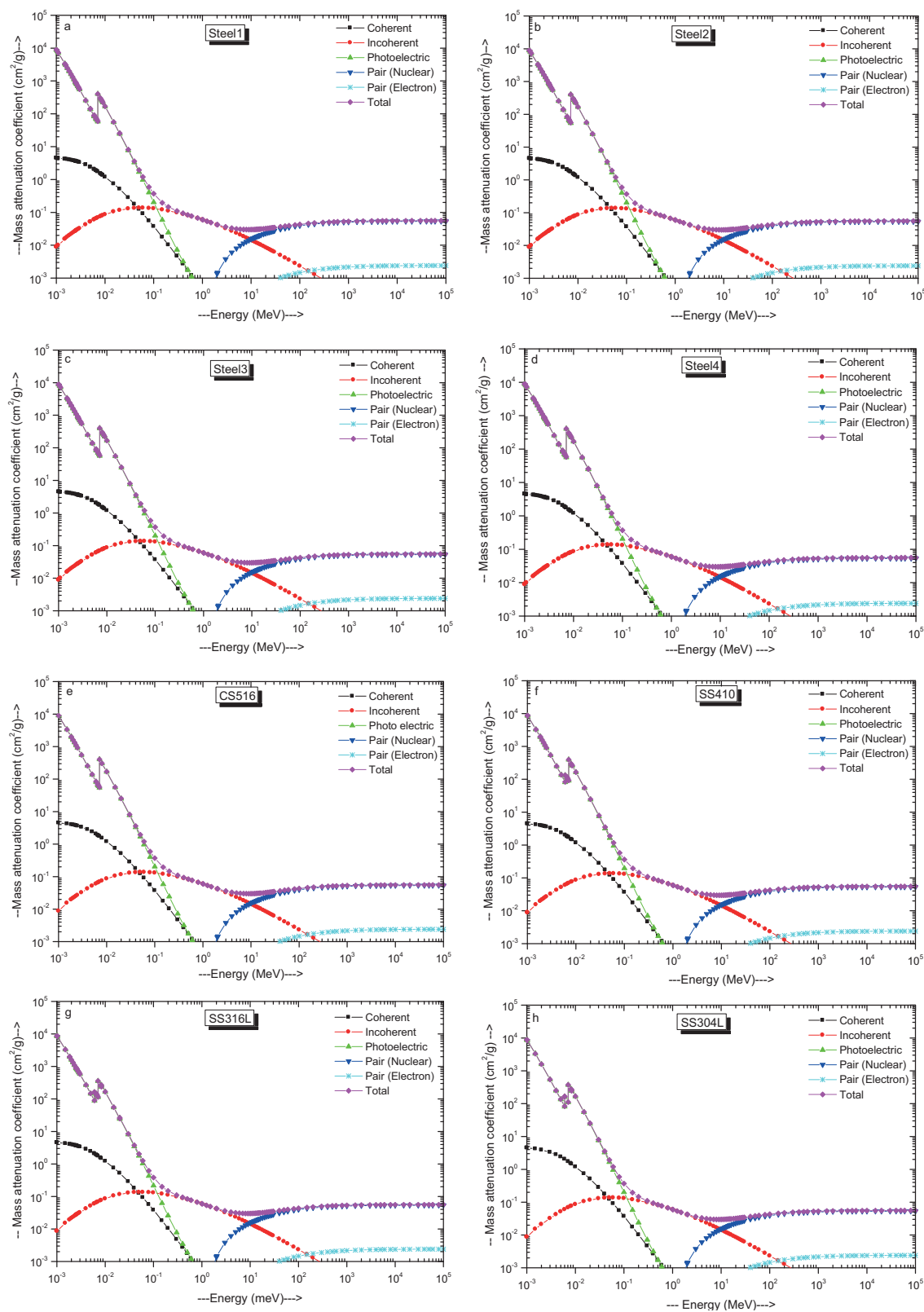

Figure 2 (a-h) - Variation in mass attenuation coefficients for partial photon interactions viz. photoelectric, Compton and pair production for carbon steel and stainless steels. 


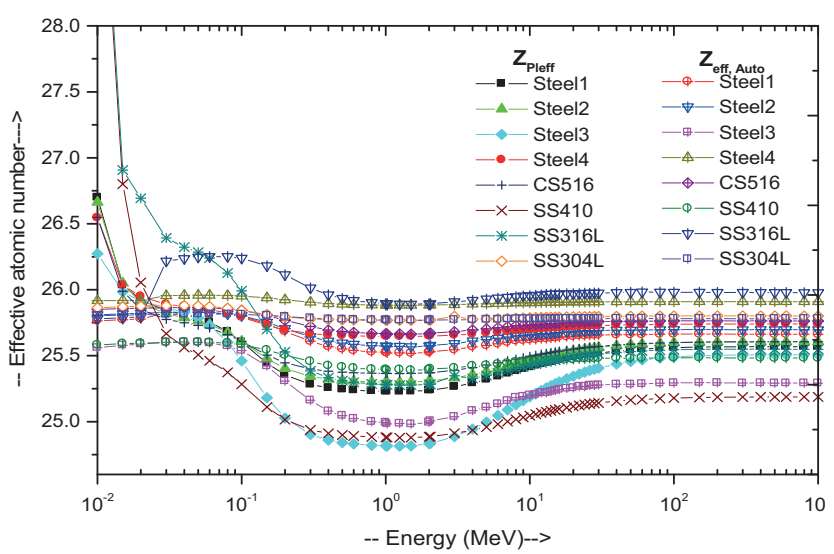

Figure 3 - Variation in effective atomic numbers of carbon steel and stainless steels with photon energy for the practical formula and robust method.

be due to the fact that the cross-section of the pair production process, in the nuclear field, is $Z^{2}$-dependent, whereas for triplet production, the cross-section varies linearly with $\mathrm{Z}$. It is also observed from Figure $2(\mathrm{a}-\mathrm{h})$ that although the pair production process initiates from $1.022 \mathrm{MeV}$, it requires more incident photon energy to overcome Compton scattering and to become the dominant photon interaction process. For the all selected steels, $\mu_{\text {steel, pp }}$ becomes equivalent to $\mu_{\text {steel, Compton }}$ at about $20 \mathrm{MeV}$ photon energy.

Finally, it is concluded that the Compton scattering dominant region is from

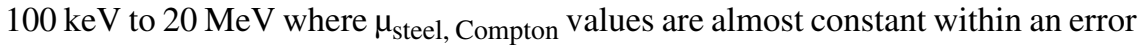
of $10 \%$ due to linear dependency on $\mathrm{Z}$. At low photon energy $(<100 \mathrm{keV})$ the variation is found to be very large in the photon energy and atomic number of constituent elements.

\subsection{Effective atomic numbers}

Variation in $Z_{\text {pleff }}$ for total photon interaction with incident photon energy is shown in Figure 3 . The variation in $\mathrm{Z}_{\text {pleff }}$ of the steels can be explained by the photo-electric absorption, Compton scattering and pair production process discussed earlier. In the photon energy range $10 \mathrm{keV}$ to $100 \mathrm{KeV}, \mathrm{Z}_{\text {pleff }}$ values of all the steels are found to be approximately constant, whereas a very large variation in $\mathrm{Z}_{\text {pleff }}$ values is observed. As a photon crosses photo-absorption and reaches the Compton region the $Z_{\text {pleff }}$ value becomes constant. Above $20 \mathrm{MeV}$ photon energy, the $\mathrm{Z}_{\text {pleff }}$ starts increasing and becomes invariable at $100 \mathrm{MeV}$ energy. It can be 


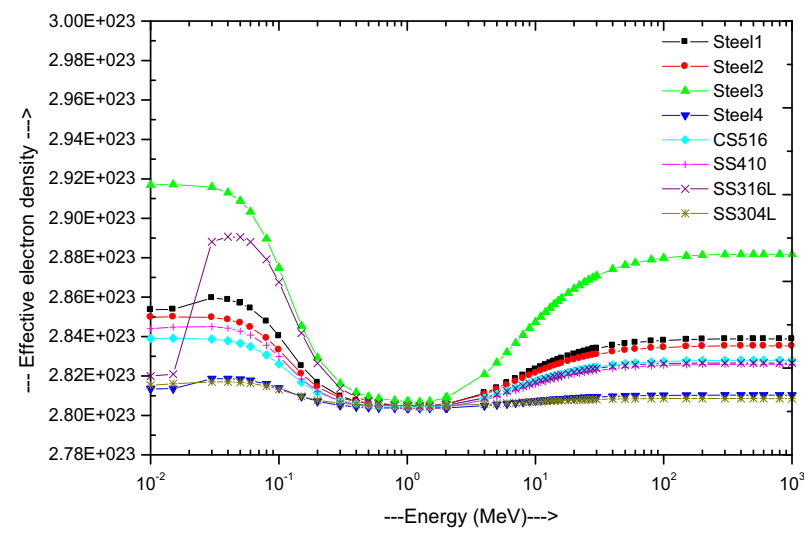

Figure 4 - Variation in effective electron densities of carbon steel and stainless steels with photon energy.

observed that the variation in $\mathrm{Z}_{\text {pleff }}$ in the Compton region is very narrow, i.e. 24.8 to 25.8 , which is due to a small variation in the weight fraction of the constituent elements. The maximum value of $\mathrm{Z}_{\text {pleff }}$ for $\mathrm{SS} 304 \mathrm{~L}$ was in the Compton scattering process and pair-production regions, whereas it was minimum for Steel 3. The variation in $\mathrm{Z}_{\text {pleff }}$ values can be explained by the contribution of elements in the steel (which is Z-dependent).

A high value of $\mathrm{Z}_{\text {pleff }}$ of steels is expected below $10 \mathrm{keV}$ photon energy due to absorption at the k-edge of the constituent elements. Except for the SS304L steel, all the others have $\mathrm{Al}, \mathrm{Si}, \mathrm{P}$ and $\mathrm{S}$ elements, for which k-edge absorption is at $1.56 \mathrm{keV}, 1.839 \mathrm{keV}, 2.145 \mathrm{keV}$ and $2.472 \mathrm{keV}$. In the case of SS304L steel, k-edge absorption appears at $5.989 \mathrm{keV}, 7.112 \mathrm{keV}$ and $8.333 \mathrm{keV}$ for $\mathrm{Cr}, \mathrm{Fe}$ and $\mathrm{Ni}$.

The effective atomic numbers calculated by $\mathrm{Z}_{\text {pleff }}$ and the Auto- $\mathrm{Z}_{\text {eff }}$ program are shown in Figure 3. From Figure 3, it can be seen that there is small variation in the estimations in the Compton scattering region, whereas there is large variation in the photo-electric absorption region. The $\mathrm{Z}_{\text {pleff }}$ values are higher than those of $\mathrm{Z}_{\text {eff, auto }}$ in the photo-electric region, whereas there is the reverse behavior in the Compton region. SS304L shows equal values of the effective atomic number for the entire photon energy region $10 \mathrm{keV}$ to $1 \mathrm{GeV}$. The $\mathrm{Z}_{\text {pleff }}$ values of SS410 and $\mathrm{SS} 316 \mathrm{~L}$ are found to be very high in the photo-electric absorption region.

\subsection{Electron densities}

Figure 4 shows the variation in $\mathrm{N}_{\text {eff }}$ with incident photon energy for all the selected steels. Among the selected steels, steel 1 shows the maximum value for electron 
density, whereas the minimum value for electron density was observed for SS304L in the $1 \mathrm{keV}$ to $1 \mathrm{GeV}$ energy region. The variation in electron density with incident photon energy follows the reciprocal trend, as observed for the total photon interaction effective atomic number. Under the dominance of any photon interaction, electron density remains constant for particular steels. However, a large variation in electron density is observed when the interaction process changes.

\subsection{Comparison with experimental results}

The experimental investigations of the effective atomic numbers of the selected steels were carried out for some photon energies in the Compton scattering region. The effective atomic numbers for SS316L and CS516 stainless steels were experimentally investigated at $662 \mathrm{keV}, 1173 \mathrm{keV}$ and $1132 \mathrm{keV}$ photon energies by ${ }^{137} \mathrm{Cs}$ and ${ }^{60} \mathrm{Co}$ sources (Fakarudin et al., 2011). Differential incoherent scattering cross-sections and the effective atomic numbers of steel alloys were carried out in the energy region $279.1 \mathrm{keV}$ to $1115.5 \mathrm{keV}$ by ${ }^{203} \mathrm{Hg},{ }^{65} \mathrm{Zn}$ and ${ }^{137} \mathrm{Cs}$ sources at scattering angles of $60^{\circ}, 80^{\circ}$ and $100^{\circ}$ (Prasanna and Kumar, 2012). It is found that the steel samples 1 to 4 are characterized by a mean effective atomic number of 25.85 for Compton scattering. It is also found that the variation in effective atomic numbers for $60^{\circ}, 80^{\circ}$ and $100^{\circ}$ is insignificant as well as almost independent of incident photon energy. Slight differences in experimental effective atomic numbers and the present study may be due to non-consideration of the molecular, chemical and crystalline environment of atoms in the mixture rule. The experimental values of effective atomic numbers were compared with the $Z_{\text {pleff }}$ of the present study and were found to be in good agreement, as given in Table II.

\subsection{Uncertainties in results}

The mass attenuation coefficients of the steels were evaluated by the weighted sum of the mass attenuation coefficients of the elements. However, the molecular, chemical or crystalline environment of the atom changes the atomic wave function. This change in wave function limits the mixture rule applications. With the exception of fine-structure regions above the absorption edge, for photon energies above $10 \mathrm{keV}$, errors from these sources are expected to be less than a few percent. In the Compton region $(10 \mathrm{keV}$ to $1 \mathrm{MeV})$ the uncertainty in mass attenuation coefficients is expected to be less than 1 to $2 \%$ because of partitioning of experimental totals into photo effect and scattering totals. At medium energies ( 1 to $100 \mathrm{MeV}$ ) uncertainty of 2 to $3 \%$ is expected because of uncertainties in the pair production cross-section, particularly the 10 to $30 \mathrm{MeV}$ photonuclear giant 
TABLE II

Comparison of effective atomic numbers from the present study with experimental values.

\begin{tabular}{cccccccc}
\hline \multirow{2}{*}{ Description } & Literature & \multicolumn{7}{c}{ Energy $(\mathrm{keV})$} \\
\cline { 3 - 8 } & & 279.1 & 661.6 & 662 & 1115.5 & 1173 & 1332 \\
\hline \multirow{2}{*}{ Steel 1 } & b & 25.90 & 25.90 & - & 25.90 & - & - \\
& c & 25.29 & 25.24 & 25.24 & 25.23 & 25.23 & 25.22 \\
Steel 2 & b & 25.80 & 26.00 & - & 26.00 & - & - \\
& c & 25.35 & 25.31 & 25.31 & 25.30 & 25.29 & 25.29 \\
Steel 3 & b & 25.80 & 26.00 & - & 25.90 & - & - \\
& c & 24.92 & 24.83 & 24.83 & 24.81 & 24.81 & 24.81 \\
Steel 4 & b & 25.90 & 25.90 & - & 25.90 & - & - \\
& c & 25.67 & 25.65 & 25.65 & 25.65 & 25.63 & 25.64 \\
SS316L & a & - & - & 26.61 & - & 26.85 & 26.88 \\
& c & 25.40 & 25.29 & 25.29 & 25.28 & 25.27 & 25.27 \\
CS516 & a & - & - & 25.66 & - & 25.89 & 26.03 \\
& c & 25.41 & 25.37 & 25.37 & 25.36 & 25.35 & 25.36 \\
\hline
\end{tabular}

a. Fakarudin et al., 2011

b. Prasanna and Kumar, 2012

c. Present study

resonance region. Above $100 \mathrm{MeV}$ the uncertainty is expected to be 1 to $2 \%$. The uncertainty in mass attenuation coefficients is expected to more than double below $10 \mathrm{keV}$ photon energy. The investigation values in our present study are very useful, with an acceptable range of uncertainties, as the steel will be used at photon energies of $5 \mathrm{keV}$ and above. In the case of the Auto- $Z_{\text {eff }}$ software, the uncertainties in the effective atomic number are $1-2 \%$ at higher energies away from absorption edges.

\section{Conclusions}

The present study provides the values of the gamma-ray mass attenuation coefficients, effective atomic numbers and electron densities of carbon steel and stainless steels. Our results show that the $\mu / \rho, Z_{\text {pleff }}$ and $N_{\text {eff }}$ values of the alloys are photon energy- and composition element-dependent and vary mainly in the photo-electric absorption and Compton scattering regions. In the photo-electric absorption region low energy $(<10 \mathrm{keV})$, very high values of $\mathrm{Z}_{\text {pleff }}$ are observed. These high values of $Z_{\text {pleff }}$ require further experimental investigations.

Acknowledgments. The authors are grateful to Professor L. Gerward for providing the WinXcom program. 


\section{REFERENCES}

Akkurt I. (2007) Effective atomic numbers for Fe-Mn alloy using transmission experiment, Chin. Phys. Lett. 24 (10), 2812-2814.

Akkurt I. (2009) Effective atomic and electron numbers of some steels at different energies, Ann. Nucl. Energy 36 (11-12), 1702-1705.

Bastug A., Gurol A., Icell O., Yusuf S. (2010) Effective atomic numbers of some composite mixutes including borax, Ann. Nucl. Energy 37, 927-933.

Berger M.J., Hubbell J.H. (1987/99) XCOM: Photon Cross Sections Database, Web Version 1.2, available at http://physics.nist.gov/xcom, National Institute of Standards and Technology, Gaithersburg, MD 20, 899, USA.

Demir D., Turşucu A., Oznülüer T. (2012) Studies on mass attenuation coefficient, effective atomic number and electron density of some vitamin, Radiat. Environ. Biophys. 51 (4), 469-475.

El-Kateb A.H., Rizk R.A.M., Abdul-Kader A.M. (2000) Determination of atomic cross-sections and effective atomic numbers for some alloys, Ann. Nucl. Energy 27, 1333-1343.

Fakarudin A.R.F., M. Iqbal S., Nor Pa'iza M.H., Ismail M. (2011) Mass attenuation coefficients, effective atomic numbers and electron densities of stainless steel and carbon steel with different energies, Journal Sains Nuklear Malaysia 23 (2), 19-25.

Gerward L., Guilbert N., Jensen K.B., Levring H. (2001) X-ray absorption in matter. Reengineering XCOM, J. Radiat. Phys. Chem. 60, 23-24.

Gerward, L., Guilbert N., Jensen K.B., Levring H. (2004) WinXcom-a program for calculating X-ray attenuation coefficients, J. Radiat. Phys. Chem. 71, 653-654.

Han I., Demir L. (2009) Determination of mass attenuation coefficients, effective atomic and electron numbers for $\mathrm{Cr}, \mathrm{Fe}$ and $\mathrm{Ni}$ alloys at different energies, Nucl. Instrum. Methods B 267, 3-8.

Hine G.J. (1952) Use wildcard to add more than one file at a time, Phys. Rev. 85, 752-755.

Hubbell J.H., Veigele W.M., Briggs E.A., Brown R.T., Cromer D.T., Howerton R.J. (1975) Atomic form factors, incoherent scattering functions, and photon scattering cross sections, J. Phys. Chem. Ref. Data 4 (3), 471-538.

İçelli O., Salih E., İsmail H.K., Güven Ç. (2005) Effective atomic numbers for CoCuNi alloys using transmission experiment, J. Quant. Spectrosc. Radiat. Trans. 91, 485-491.

Kaewkhao J., Laopaiboon J., Chewpraditkul W. (2008) Determination of effective atomic numbers and effective electron densities for Cu/Zn alloy, J. Quant. Spectrosc. Radiat. Trans. 109, 1260-1265.

Manohara S.R., Hanagodimath S.M., Thind K.S., Gerward L. (2008) On the effective atomic number and electron density: A comprehensive set of formulas for all types of materials and energies above $1 \mathrm{keV}$, Nucl. Instrum. Meth. B 266, 3906-3912.

Medhat M.E. (2011) Studies on effective atomic numbers and electron densities in different solid state track detectors in the energy range $1 \mathrm{keV}-100 \mathrm{GeV}$, Ann. Nucl. Energy 38 (6), 1252-1263.

Michael E.W., Tyler B.C. (2011) Atomic weight of elements 2009, IUPAC Technical Report, Pure Appl. Chem. 83 (2), 359-396.

Murty V.R.K., Winkoun D.P., Devan K.R.S. (2000) Effective atomic numbers for W/Cu alloy using transmission experiments, Appl. Radiat. Isotopes 53, 945-948.

Prasad S.G., Parthasaradhi K., Bloomer W.D. (1998) Effective atomic numbers for photoabsorption in alloys in the energy region of absorption edges, Radiat. Phys. Chem. 53, 449-453.

Prasanna S.K., Kumar T.K. (2012) Differential incoherent scattering cross sections and effective atomic numbers of steels alloys in the energy region $279.1 \mathrm{keV}$ to $1115.5 \mathrm{keV}$. In: Proceedings NSRP-19, Dec 12-14, Mamallapuram, Tamilnadu, India, pp. 28-30. 
Shivaliga G., Krishnaveni S., Yashoda, Umesh T.K., Ramakrishna G. (2004) Mass attenuation coefficients, effective atomic number and electron densities of thermolumininescent dosimetric compounds, PRAMANA 63 (3), 529-541.

Singh V.P., Badiger N.M. (2012a), Photon interaction parameters of some spin ice compound materials, Int. J. Nuclear Energy Science and Technology 7 (1), 57-74.

Singh V.P., Badiger N.M. (2012b) Effective atomic numbers, electron densities and tissue equivalence of some gases and mixtures for dosemetry of radiation detectors, Nuclear Technology \& Radiation Protection 27 (2), 117-124.

Singh V.P., Badiger N.M. (2013) Study of Effective Atomic Numbers and Electron Densities, Kerma of Alcohols, Phantom and Human Organs and Tissues Substitutes, Nuclear Technology \& Radiation Protection (accepted for publication).

Taylor M.L., Smith R.L., Dossing F., Franich R.D. (2012) Robust calculation of effective atomic numbers: The Auto- $\mathrm{Z}_{\mathrm{eff}}$ software, Med. Phys. 39, 1769-1778.

Woods J. (1982) Computational Methods in Reactor Shielding, Pergamon Press. 